\title{
Late incidence and predictors of persistent or recurrent heart failure in patients with aortic prosthetic valves
}

Marc Ruel, MD, MPH ${ }^{a, b}$

Fraser D. Rubens, $\mathrm{MD}^{\mathrm{a}}$

Roy G. Masters, MD

Andrew L. Pipe, MDa

Pierre Bédard, $M D^{\mathrm{a}}$

Paul J. Hendry, MD

B. Khanh Lam, MD, MPH

Ian G. Burwash, $M^{\mathrm{c}}$

William G. Goldstein, MD

Maurice P. Brais, MD

Wilbert J. Keon, MD

Thierry G. Mesana, MD, PhDa
From the Division of Cardiac Surgery, ${ }^{a}$ the Department of Epidemiology, ${ }^{\mathrm{b}}$ and the Division of Cardiology, ${ }^{c}$ University of Ottawa, Ottawa, Ontario, Canada.

Read at the Eighty-third Annual Meeting of The American Association for Thoracic Surgery, Boston, Mass, May 4-7, 2003.

Received for publication May 2, 2003; revisions requested June 20, 2003; revisions received July 21, 2003; accepted for publication Sept 11, 2003.

Address for reprints: Marc Ruel, MD, MPH, University of Ottawa Heart Institute, 40 Ruskin St, Ste 3403, Ottawa, Ontario, Canada K1Y 4W7 (E-mail: mruel@ ottawaheart.ca).

J Thorac Cardiovasc Surg 2004;127:149-59 $0022-5223 / \$ 30.00$

Copyright $\odot 2004$ by The American Association for Thoracic Surgery

doi:10.1016/j.jtcvs.2003.07.043
Background: We examined factors associated with persistent or recurrent congestive heart failure after aortic valve replacement.

Methods: Patients who underwent aortic valve replacement with contemporary prostheses $(n=1563)$ were followed up with annual clinical assessment and echocardiography. The effect of demographic, comorbid, and valve-related variables on the composite outcome of New York Heart Association class III or IV symptoms or congestive heart failure death after surgery was evaluated with stratified log-rank tests, Cox proportional hazard models, and logistic regression. Factors associated with all-cause death were also examined. Prediction models were bootstrapped 1000 times.

Results: Total follow-up was 6768 patient-years (mean, $4.3 \pm 3.3$ years; range, 60 days to 17.1 years). Freedom from congestive heart failure or congestive heart failure death was $98.6 \% \pm 0.3 \%, 88.6 \% \pm 1.0 \%, 73.9 \% \pm 2.3 \%$, and $45.2 \% \pm$ $8.5 \%$ at $1,5,10$, and 15 years, respectively. Age, preoperative New York Heart Association class, left ventricular grade, atrial fibrillation, coronary artery disease, smoking, and redo status predicted congestive heart failure after surgery (all $P<$ .05). Larger prosthesis size and effective orifice area, both absolute and indexed for body surface area, were independently associated with freedom from congestive heart failure. Increased transprosthesis gradients were predicted by prosthesispatient mismatch and were associated with congestive heart failure after surgery. Mismatch defined as an effective orifice area/body surface area of $0.80 \mathrm{~cm}^{2} / \mathrm{m}^{2}$ or less was a significant predictor of congestive heart failure events after surgery, but mismatch defined as an effective orifice area/body surface area of $0.85 \mathrm{~cm}^{2} / \mathrm{m}^{2}$ or less was not. Small prosthesis size and mismatch were not significantly associated with all-cause mortality.

Conclusions: These analyses identify independent predictors of congestive heart failure symptoms and congestive heart failure death late after aortic valve replacement and indicate that prosthesis size has a significant effect on this cardiac end point, but not on overall survival after aortic valve replacement. 


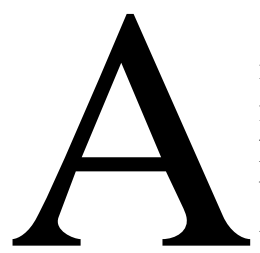

common goal of heart valve replacement is to prevent or cure symptoms of heart failure and decrease the likelihood of heart failure-related death. An extensive body of literature has defined the optimal timing of valve replacement on the basis of symptoms and cardiac function to achieve this goal in the highest proportion of patients while minimizing the effects of operation and prosthesis-related complications on survival and quality of life. ${ }^{1}$

Few studies have systematically examined the effects of patient- and prosthesis-related characteristics on the occurrence of heart failure after valve replacement. Surgeons and cardiologists alike know that heart failure is not always cured or prevented by valve replacement. ${ }^{1}$ Knowing the risk factors for heart failure after valve replacement could prove useful for determining a patient's postoperative prognosis and assist in clinical decision making.

One factor of interest is the ability of the replacement prosthesis to allow unimpeded blood flow. This parameter is potentially modifiable and is related to the size and type of prosthesis implanted at operation. Many surgeons intuitively perceive that prosthesis size and type may influence long-term clinical outcome after aortic valve replacement. Expectations may be hampered, however, by technical limitations imposed by the size of the native annulus after excision of the diseased valve. In patients with a small native aortic annulus, a prosthesis with a larger effective orifice area (EOA) may be implanted by performing root enlargement ${ }^{2}$ or root replacement ${ }^{3}$ or by using a stentless valve, ${ }^{4,5}$ but these techniques are surgically more challenging and may be associated with increased morbidity, ${ }^{6,7}$ particularly in inexperienced hands.

The approach to this dilemma remains controversial. Recent data suggest that the long-term consequences of small aortic prosthesis implantation may be less than anticipated with respect to all-cause mortality, thus suggesting that complex alternative procedures may be unnecessary. ${ }^{8}$ Other data suggest that accepting this predicament without correction may lead to placement of a small prosthesis with resultant impediment of blood flow and less recovery of left ventricular function, which could affect quality of life and freedom from cardiac death. ${ }^{9}$ For these reasons, we aimed at elucidating whether aortic prosthesis size, among other factors, has an independent effect on freedom from postoperative heart failure.

This study, therefore, examines the effect of patient- and prosthesis-related factors on the occurrence of clinical heart failure after surgery in a cohort of patients followed up after aortic valve replacement.

\section{Methods}

\section{Patients and Follow-up}

Adult patients ( $\geq 18$ years of age) who underwent aortic valve replacement between 1976 and 2001 at the University of Ottawa
TABLE 1. Prevalence and mean values of preoperative variables, by type of prosthesis

\begin{tabular}{lcc}
\hline Variable & $\begin{array}{c}\text { Mechanical } \\
\text { (n = 873) }\end{array}$ & $\begin{array}{c}\text { Bioprosthetic } \\
\text { (n = 690) }\end{array}$ \\
\hline Female sex & $30.6 \%$ & $34.8 \%$ \\
Age at operation (y) & $59.7 \pm 11.9$ & $68.9 \pm 13.3^{*}$ \\
Body surface area $\left(\mathrm{m}^{2}\right)$ & $1.85 \pm 0.23$ & $1.86 \pm 0.26$ \\
NYHA class & & \\
I & $25.2 \%$ & $27.7 \%$ \\
II & $33.3 \%$ & $27.5 \%$ \\
III & $26.4 \%$ & $29.8 \%$ \\
IV & $15.1 \%$ & $15.0 \%$ \\
Left ventricular grade & & \\
1 & $41.7 \%$ & $55.0 \% *$ \\
2 & $25.6 \%$ & $21.7 \%$ \\
3 & $21.1 \%$ & $15.5 \%$ \\
4 & $11.6 \%$ & $7.8 \%$ \\
LVEDP (mm Hg) & $21.5 \pm 9.1$ & $18.9 \pm 7.9$ \\
Coronary artery disease & $33.9 \%$ & $37.1 \%$ \\
Chronic atrial fibrillation & $5.2 \%$ & $3.3 \%$ \\
Previous aortic valve replacement & $21.8 \%$ & $6.4 \% *$ \\
Operative indication & & \\
Predominant stenosis & $51.6 \%$ & $74.9 \% *$ \\
Predominant insufficiency & $39.1 \%$ & $15.3 \% *$ \\
\hline
\end{tabular}

Values are reported as mean \pm SD or $\%$.

LVEDP, Left ventricular end-diastolic pressure; NYHA, New York Heart Association.

${ }^{*} P<.05$ versus patients with mechanical valves.

Heart Institute were followed up annually in a dedicated valve clinic. All patients had a history focused on the determination of functional status and the occurrence of valve-related complications, physical examination, electrocardiogram, chest radiograph, complete blood count, serum chemistries, and international normalized ratio determinations when applicable. Because of the potential effect of the prosthesis on the outcome of interest, patients who received valves that have since been withdrawn from the North American market, such as the IonescuShiley (Shiley, Inc, Irvine, Calif) or Medtronic Intact (Minneapolis, Minn) prostheses, were excluded from the analyses. The studied cohort included 1683 adult patients who underwent replacement of the aortic valve with prostheses that are still commercially available today and who survived the perioperative period. Of these 1683 patients, 119 were lost to follow-up or died during the second postoperative month, and 1563 (92.9\%) were followed up as outpatients after a minimum of 60 days after surgery.

The preoperative characteristics of the cohort are presented in Table 1. Total follow-up was 6768 patient-years, with a mean duration of $4.3 \pm 3.3$ years (range, 60 days to 17.1 years). Seventy-nine percent of patients had an average of 3.5 postoperative transthoracic echocardiograms performed on an annual basis at our institution, and these were interpreted by 1 of 4 physicians, who determined mean and peak transprosthesis gradients according to the recommendations of the American Society of Echocardiography. 


\section{Types of Prostheses}

Table 2 shows the types of valve prostheses implanted in the study population. All patients received prostheses that were still commercially available as of this writing and that were implanted and oriented according to the manufacturer's recommendations. Because of the continued availability of the St Jude Medical (St Jude Medical, Inc, St Paul, Minn), Medtronic Hall (Medtronic), and CarboMedics (Sulzer Carbomedics, Inc, Austin, Tex) mechanical valves and the discontinuation of several models of bioprostheses over the past 2 decades, a predominance of mechanical valves is found in the study cohort.

\section{Definition of Heart Failure}

Heart failure after aortic valve replacement was defined as the composite end point of (1) New York Heart Association (NYHA) functional class III or IV for more than 4 consecutive weeks or (2) death where the primary or main contributing diagnosis was congestive heart failure (CHF). Clinical impressions were corroborated with physical examination, chest radiograph, electrocardiogram, and echocardiography findings when available. Cases of primary nonstructural dysfunction resulting from severe paravalvular leaks, valve thrombosis, or endocarditis were excluded as heart failure events for the purposes of this study. Other prosthesisrelated complications were recorded according to the "Guidelines for Reporting Morbidity and Mortality After Cardiac Valvular Operations." 10,11

\section{Statistical Analyses}

Data entries. Data were imported and analyzed in Intercooled Stata version 8 (Stata, College Station, Tex). Patients were censored at the time of their last follow-up visit if they had not experienced at least 1 episode of NYHA functional class III or IV for 4 weeks or more or had died from a CHF-related event. Deaths from an unknown cause were not considered to result from CHF and were treated as a censored event if the patient had not previously experienced NYHA class III or IV symptoms. When missing data were encountered, they were not replaced or imputed, and analyses were performed with a sample size equal to the number of complete entries.

Prosthesis size. The model and manufacturer valve size of the implanted prosthesis was available for all patients. Because of variations in size nomenclature between manufacturers and models, the valve size value of each prosthesis model and size was imputed with (1) its internal diameter (based on work by Christakis and associates ${ }^{12}$ and supplemented with data provided by the valve manufacturer) and (2) its echocardiographic in vivo EOA reported in the literature from patients with normally functioning prostheses. ${ }^{13}$ If more than 1 value of in vivo EOA was published for a given prosthesis type and size, the average of the values was used.

Transprosthesis gradient. The aortic peak and mean transprosthesis gradients determined at the time follow-up echocardiography were used to examine their effect on the heart failure hazard, both as a continuous variable and as an arbitrary dichotomous parameter defined by a gradient value at or above the 90th percentile. Values from the last echocardiographic examination available before demonstration of structural valvular deterioration or recurrent heart failure symptoms were used, except in patients with persistent heart failure symptoms after operation, in whom
TABLE 2. Implanted aortic prostheses and manufacturer valve size

\begin{tabular}{lr}
\hline Variable & $\begin{array}{c}\text { Number implanted } \\
\text { (median size; range [mm]) }\end{array}$ \\
\hline Mechanical & \\
St Jude Medical standard & $274(23 ; 19-33)$ \\
St Jude Medical HP & $80(23 ; 19-27)$ \\
Medtronic Hall & $280(23 ; 20-29)$ \\
CarboMedics & $225(25 ; 21-31)$ \\
MCRI On-X & $14(23 ; 19-27)$ \\
Bioprosthetic & \\
Medtronic Hancock II & $408(23 ; 21-31)$ \\
Medtronic Hancock modified & $126(21 ; 19-29)$ \\
$\quad$ orifice & \\
Homograft & $79(22 ; 18-26)$ \\
Edwards Perimount & $60(23 ; 21-27)$ \\
Stentless porcine & $10(25 ; 21-27)$ \\
Carpentier-Edwards standard & $7(23 ; 19-31)$ \\
\hline
\end{tabular}

values from the first postoperative outpatient echocardiographic examination were used.

Cumulative incidence of heart failure. Nonparametric estimates of overall, non-risk-adjusted freedom from the composite heart failure end point over time were determined by the method of Kaplan and Meier. Risk-adjusted and stratified estimates of freedom from CHF death and all-cause death were also generated. Survival and failure rates are reported as mean \pm SEM $(95 \%$ confidence interval lower bound, $95 \%$ confidence interval upper bound).

Univariate analyses. Possible predictors for CHF were individually tested for equality of freedom from heart failure with a $\log$-rank test. Effect estimates and $P$ values were used to guide multivariate model selection and are not reported.

Multivariate analyses. For heart failure and death hazard, the proportional hazard assumption was tested with generalized CoxSnell residuals, with $-\ln [-\ln$ (survival)] probabilities, and by addressing, in the case of continuous variables, whether a significant interaction with time was present. Semiparametric Cox proportional hazards models were developed by incorporating variables that had a $P$ value of .05 or less on univariate analysis and by forcing into each model patient- and prosthesis-related variables that could constitute possible confounders regardless of their univariate $P$ value. Variables used in the models are reported in Tables 3 and 4. To account for positive or negative confounding, no automated model-selection procedure was used, and all reported variables were used simultaneously unless collinearity was present (defined as Spearman's rank correlation coefficient $\geq 0.30$ and $P$ value $<.005$ between 2 variables). Each model was evaluated with a score test and rejected if $P$ was 0.05 or higher. The effect of prosthesis size measures on the cumulative incidence of heart failure symptoms and CHF-related death at 1,5 , and 10 years was examined with logistic regression by using the same covariates as in the Cox proportional hazards models.

Bootstrapped simulations. Each proportional hazards model was subjected to 1000 bootstrap replications by using random samples generated from and consisting of the same number of patients as the original model. ${ }^{14}$ Each simulation sample was 
TABLE 3. Predictors of NYHA class III or IV symptoms or CHF-related death

\begin{tabular}{|c|c|c|c|}
\hline Variable & $\begin{array}{c}\text { Adjusted } \\
\text { hazard ratio }\end{array}$ & $95 \% \mathrm{Cl}$ & $P$ value \\
\hline \multicolumn{4}{|l|}{ Significant independent predictors } \\
\hline Age $>65$ y & 1.62 & $1.07,2.44$ & .022 \\
\hline Atrial fibrillation & 3.18 & $1.71,5.92$ & $<.001$ \\
\hline Preoperative NYHA class* & 1.38 & $1.14,1.67$ & .001 \\
\hline $\begin{array}{l}\text { Preoperative left ventricular } \\
\text { grade* }\end{array}$ & 1.74 & $1.30,2.33$ & $<.001$ \\
\hline $\begin{array}{l}\text { Preoperative diastolic } \\
\text { pulmonary artery pressure } \\
(\text { per } \mathrm{mm} \mathrm{Hg})^{*}\end{array}$ & 1.01 & $1.00,1.02$ & .030 \\
\hline Coronary artery disease & 1.55 & $2.11,5.12$ & .031 \\
\hline Smoking & 3.29 & $1.85,3.60$ & $<.001$ \\
\hline Redo aortic valve replacement & 1.67 & $1.15,2.42$ & .007 \\
\hline Tricuspid insufficiency & 3.61 & $1.09,12.0$ & .036 \\
\hline $\begin{array}{l}\text { Manufacturer valve size (per } 1 \\
\text { size increase) } \dagger\end{array}$ & 0.81 & $0.67,0.99$ & .037 \\
\hline $\begin{array}{l}\text { Internal valve diameter (per } \\
\mathrm{mm}) \dagger\end{array}$ & 0.90 & $0.82,0.99$ & .046 \\
\hline $\begin{array}{l}\text { In vivo effective orifice area } \\
\left(\text { per } \mathrm{cm}^{2}\right) \dagger\end{array}$ & 0.41 & $0.17,0.97$ & .042 \\
\hline $\begin{array}{l}\text { Mismatch defined as } \leq 0.75 \\
\mathrm{~cm}^{2} / \mathrm{m}^{2} \dagger \ddagger\end{array}$ & 1.64 & $1.01,2.56$ & .047 \\
\hline $\begin{array}{l}\text { Mismatch defined as } \leq 0.80 \\
\mathrm{~cm}^{2} / \mathrm{m}^{2} \dagger \ddagger\end{array}$ & 1.61 & $1.01,2.57$ & .044 \\
\hline $\begin{array}{l}\text { Peak transprosthesis gradient } \\
\text { (per } \mathrm{mm}) \dagger\end{array}$ & 1.03 & $1.02,1.05$ & $<.001$ \\
\hline $\begin{array}{l}\text { Mean transprosthesis gradient } \\
\text { (per mm) } \dagger\end{array}$ & 1.06 & $1.03,1.09$ & $<.001$ \\
\hline $\begin{array}{l}\text { Peak transprosthesis gradient } \\
\quad \geq 90 \text { th percentile } \dagger\end{array}$ & 2.48 & $1.32,4.68$ & .005 \\
\hline $\begin{array}{l}\text { Mean transprosthesis gradient } \\
\geq 90 \text { th percentile } \dagger\end{array}$ & 2.84 & $1.49,5.40$ & .001 \\
\hline \multicolumn{4}{|c|}{ Nonsignificant variables included in the model } \\
\hline $\begin{array}{l}\text { Mismatch defined as } \leq 0.85 \\
\mathrm{~cm}^{2} / \mathrm{m}^{2} \dagger \ddagger\end{array}$ & 1.20 & $0.79,1.82$ & .40 \\
\hline Body surface area & 1.50 & $0.68,3.28$ & .31 \\
\hline Diabetes mellitus & 1.02 & $0.50,2.10$ & .95 \\
\hline History of stroke & 0.94 & $0.12,7.45$ & .95 \\
\hline $\begin{array}{l}\text { Bioprosthetic (vs mechanical) } \\
\text { valve }\end{array}$ & 0.67 & $0.43,1.06$ & .09 \\
\hline $\begin{array}{l}\text { Operative indication for } \\
\text { predominant } \mathrm{Al}\end{array}$ & 0.99 & $0.61,1.61$ & .97 \\
\hline
\end{tabular}

$\overline{A l \text {, Aortic insufficiency; NYHA, New York Heart Association; } C l \text {, confidence }}$ interval.

* + Collinear variables that were successively but not simultaneously included in the model.

¥Models that included mismatch terms (determined by the ratio of the prosthesis's in vivo effective orifice area divided by the patient's body surface area) did not also incorporate body surface area as an additional term.

analyzed as a new patient population, and coefficients of the models were adjusted for confounding. Bootstrap estimates of SE, bias, and $95 \%$ confidence intervals were calculated for each covariate by using a bias-corrected method derived from the 1000 replications.
TABLE 4. Predictors of all-cause death

\begin{tabular}{|c|c|c|c|}
\hline Variable & $\begin{array}{c}\text { Adjusted } \\
\text { hazard ratio }\end{array}$ & $95 \% \mathrm{Cl}$ & $P$ value \\
\hline \multicolumn{4}{|l|}{ Significant independent predictors } \\
\hline Age (per year) & 1.05 & $1.02,1.07$ & $<.001$ \\
\hline Atrial fibrillation & 2.39 & $1.10,5.18$ & .028 \\
\hline Preoperative NYHA class* & 1.24 & $1.01,1.52$ & .037 \\
\hline $\begin{array}{l}\text { Preoperative left ventricular } \\
\text { grade* }\end{array}$ & 1.41 & $1.00,1.99$ & .048 \\
\hline $\begin{array}{l}\text { Preoperative diastolic pulmonary } \\
\text { artery pressure (per mm } \mathrm{Hg})^{*}\end{array}$ & 1.01 & $1.00,1.02$ & .030 \\
\hline Coronary artery disease & 1.62 & $1.05,2.50$ & .029 \\
\hline $\begin{array}{l}\text { Number of pack-years smoked } \\
\text { (per pack-year) }\end{array}$ & 1.02 & $1.01,1.04$ & .006 \\
\hline $\begin{array}{l}\text { Insulin-dependent diabetes } \\
\text { mellitus }\end{array}$ & 3.32 & $1.02,11.2$ & .047 \\
\hline \multicolumn{4}{|c|}{ Nonsignificant variables included in the model } \\
\hline Redo aortic valve replacement & 1.10 & $0.62,1.97$ & .74 \\
\hline Tricuspid insufficiency & 1.13 & $0.15,8.42$ & .90 \\
\hline $\begin{array}{l}\text { Manufacturer valve size (per } 1 \\
\text { size increase) } \dagger\end{array}$ & 1.00 & $0.81,1.23$ & .97 \\
\hline $\begin{array}{l}\text { Internal valve diameter (per } \\
\mathrm{mm})^{*}\end{array}$ & 1.02 & $0.92,1.13$ & .77 \\
\hline $\begin{array}{l}\text { In vivo effective orifice area (per } \\
\left.\mathrm{cm}^{2}\right)^{*}\end{array}$ & 0.41 & $0.15,1.14$ & .09 \\
\hline $\begin{array}{l}\text { Mismatch defined as } \leq 0.75 \mathrm{~cm}^{2} / \\
\mathrm{m}^{2 *}+\end{array}$ & 1.27 & $0.77,2.07$ & .34 \\
\hline $\begin{array}{l}\text { Mismatch defined as } \leq 0.80 \mathrm{~cm}^{2} / \\
\mathrm{m}^{2 *} \dagger\end{array}$ & 1.42 & $0.88,2.31$ & .15 \\
\hline $\begin{array}{l}\text { Mismatch defined as } \leq 0.85 \mathrm{~cm}^{2} / \\
\mathrm{m}^{2 *} \dagger\end{array}$ & 1.34 & $0.88,2.02$ & .17 \\
\hline Body surface area & 1.32 & $0.57,3.04$ & .52 \\
\hline $\begin{array}{l}\text { Bioprosthetic (vs mechanical) } \\
\text { valve }\end{array}$ & 1.10 & $0.70,1.73$ & .69 \\
\hline $\begin{array}{l}\text { Operative indication for } \\
\text { predominant } \mathrm{Al}\end{array}$ & 1.01 & $0.58,1.78$ & .96 \\
\hline
\end{tabular}

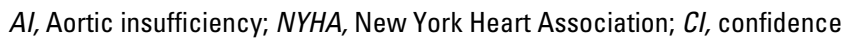
interval.

${ }^{*}$ Collinear variables that were successively but not simultaneously included in the model.

tModels that included mismatch terms (determined by the ratio of the prosthesis's in vivo effective orifice area divided by the patient's body surface area) did not also incorporate body surface area as an additional term.

\section{Results}

\section{Cumulative Incidence of Heart Failure Events After Valve Replacement}

Freedom from the composite outcome of NYHA functional class III or IV symptoms or CHF death after aortic valve replacement was $98.6 \% \pm 0.3 \%, 88.6 \% \pm 1.0 \%, 73.9 \% \pm$ $2.2 \%$, and $45.2 \% \pm 8.5 \%$ at $1,5,10$, and 15 years, respectively. Figure 1 shows the freedom from NYHA class III or IV symptoms not leading to CHF-related death (Figure 1, $A$ ), the freedom from CHF-related death (Figure 1,B), and the freedom from all-cause death (Figure 1,C) in patients with mechanical and bioprosthetic aortic valves. 


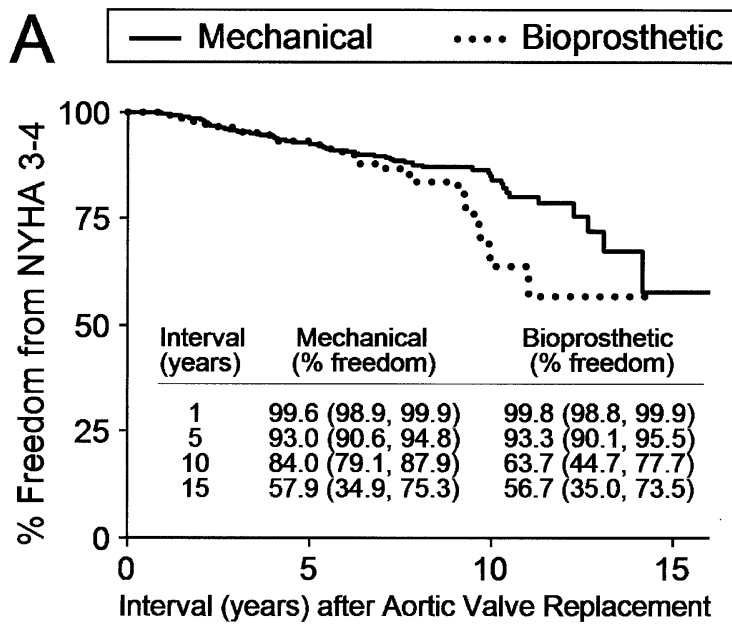

\section{At Risk:}

- 873

… 690

B

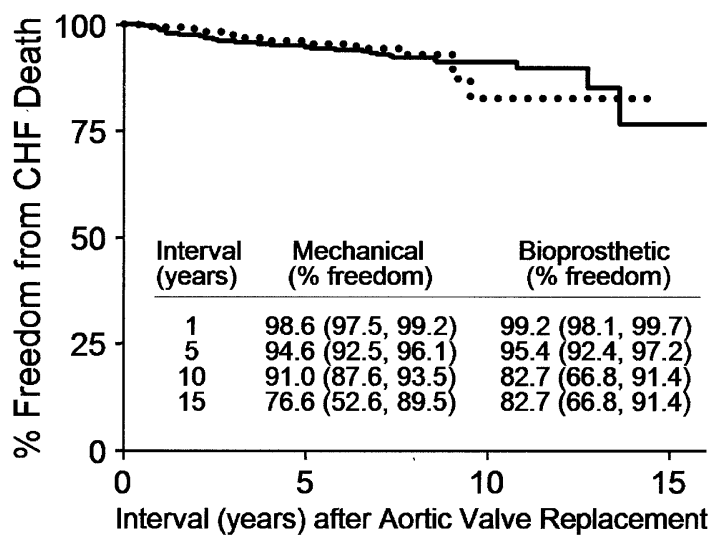

At Risk

$-873$

… 690

383

166
383

166
106

13

Figure 1. Cumulative incidence of heart failure events and death. Kaplan-Meier estimates of the freedom from NYHA class III or IV symptoms (A), CHF-related death (B), and all-cause death (C) in patients with aortic mechanical and bioprosthetic valves. ${ }^{*} P=.02$ versus patients with mechanical valves.

\section{Risk Factors for Heart Failure}

Patient-related factors. Table 3 displays the risk factors for heart failure late after aortic valve replacement. Significant independent predictors included age over 65 years (hazard ratio [HR], 1.6), atrial fibrillation (HR, 3.2), preoperative functional class (HR, 1.4 per class increase), left ventricular grade (HR, 1.7 per unit increase), pulmonary artery diastolic pressures (HR, 1.01 per millimeter increase), coronary artery disease at the time of operation (HR, 1.6),

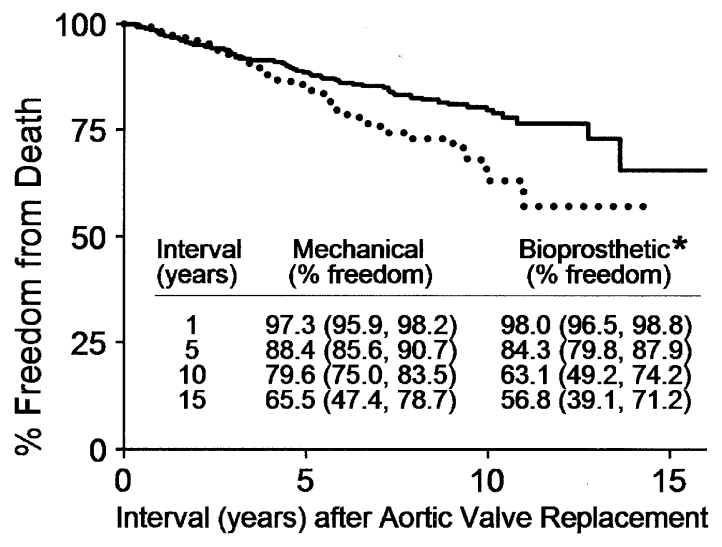

At Risk:

$\begin{array}{lllll}- & 873 & 389 & 106 & 6 \\ \ldots & 690 & 167 & 14 & 1\end{array}$


TABLE 5. Effect of aortic prosthesis size on the cumulative incidence of NYHA class III or IV or CHF death at 1, 5, and $10 \mathrm{y}$ after aortic valve replacement

\begin{tabular}{llll}
\hline Variable & \multicolumn{1}{c}{ 1-y Odds ratio } & 5-y Odds ratio & 10-y 0dds ratio \\
\hline Manufacturer valve size (per unit) & $0.72(0.54,0.98 ; 0.037)$ & $0.78(0.59,1.01 ; 0.07)$ & $0.77(0.54,1.08 ; 0.14)$ \\
Internal valve diameter (per mm) & $0.85(0.73,0.99 ; 0.043)$ & $0.92(0.80,1.05 ; 0.23)$ & $0.92(0.77,1.10 ; 0.37)$ \\
In vivo effective orifice area $(\mathrm{per} \mathrm{cm})$ & $0.40(0.18,0.87 ; 0.021)$ & $0.26(0.07,0.95 ; 0.041)$ & $0.44(0.17,1.15 ; 0.09)$ \\
Mismatch defined as $\leq 0.75 \mathrm{~cm}^{2} / \mathrm{m}^{2}$ & $2.07(0.96,4.44 ; 0.06)$ & $2.09(1.03,4.27 ; 0.043)$ & $1.98(0.71,5.51 ; 0.19)$ \\
Mismatch defined as $\leq 0.80 \mathrm{~cm}^{2} / \mathrm{m}^{2}$ & $2.45(1.17,5.12 ; 0.017)$ & $2.08(1.04,4.15 ; 0.038)$ & $1.93(0.77,4.82 ; 0.16)$ \\
Mismatch defined as $\leq 0.85 \mathrm{~cm}^{2} / \mathrm{m}^{2}$ & $1.80(0.94,3.42 ; 0.08)$ & $1.41(0.78,2.54 ; 0.26)$ & $1.48(0.69,3.16 ; 0.67)$ \\
\hline
\end{tabular}

Other variables included in the model were age over $65 \mathrm{y}$, atrial fibrillation, preoperative NYHA class, coronary disease, smoking, history of stroke, diabetes mellitus, operative indication for predominant aortic insufficiency (vs stenosis), redo aortic valve replacement, bioprosthetic (vs mechanical) valve, tricuspid insufficiency, and body surface area. Data presented as odds ratio (lower $95 \%$ confidence limit, upper $95 \%$ confidence limit; $P$ value). NYHA, New York Heart Association.

diameter (HR, 0.9 per millimeter increase), and in vivo EOA (HR, 0.6 per square centimeter increase). Each of these associations was statistically significant, regardless of whether body surface area (BSA) was simultaneously accounted for in the model.

Of 1226 patients in whom the prosthesis EOA indexed for BSA (iEOA) could be determined, 392 (32.0\%) had an iEOA at or less than $0.85 \mathrm{~cm}^{2} / \mathrm{m}^{2}, 315(25.7 \%)$ had an iEOA at or less than $0.80 \mathrm{~cm}^{2} / \mathrm{m}^{2}$, and $232(18.9 \%)$ had an iEOA at or less than $0.75 \mathrm{~cm}^{2} / \mathrm{m}^{2}$. These 3 possible definitions of the prosthesis-patient mismatch concept were examined in the multivariate models (Tables 3 and 5): mismatch defined both as iEOA less than or equal to 0.75 $\mathrm{cm}^{2} / \mathrm{m}^{2}$ and as iEOA less than or equal to $0.80 \mathrm{~cm}^{2} / \mathrm{m}^{2}$ independently predicted decreased freedom from heart failure events after aortic valve replacement (HR, 1.6), but mismatch defined as iEOA less than or equal to $0.85 \mathrm{~cm}^{2} / \mathrm{m}^{2}$ did not have a significant effect on this outcome.

The effect of aortic prosthesis size and mismatch on the cumulative incidence of CHF-related events at 1, 5, and 10 years is shown in Table 5. All measures of prosthesis size (with the exception of the internal prosthesis diameter at 5 years) predicted freedom from heart failure at 1 and 5 years after aortic valve replacement, but none had an effect at 10 years after valve replacement. Mismatch defined at both thresholds of iEOA less than or equal to $0.75 \mathrm{~cm}^{2} / \mathrm{m}^{2}$ and iEOA less than or equal to $0.80 \mathrm{~cm}^{2} / \mathrm{m}^{2}$ was predictive of a decreased freedom from heart failure at 5 years (odds ratio, 2.1), but mismatch defined at an iEOA less than or equal to $0.85 \mathrm{~cm}^{2} / \mathrm{m}^{2}$ again was not. No prosthesis size or mismatch parameter was predictive of freedom from heart failure at 10 years after valve replacement.

The effect of upper $\left(0.85 \mathrm{~cm}^{2} / \mathrm{m}^{2}\right)$ and lower $\left(0.75 \mathrm{~cm}^{2} /\right.$ $\mathrm{m}^{2}$ ) mismatch thresholds on the risk-adjusted incidence of CHF-related death is presented in Figure 2. Patients with an iEOA between 0.75 and $0.85 \mathrm{~cm}^{2} / \mathrm{m}^{2}$ had an equivalent risk of CHF-related death to those without any form of mismatch (HR 0.9 vs nonmismatch patients; $P=.790$ ); however, in patients with an iEOA of $0.75 \mathrm{~cm}^{2} / \mathrm{m}^{2}$ or less, a trend toward a higher risk of CHF-related death was observed (HR 1.8 vs nonmismatch patients; $P=.109$ ).

The median transprosthesis peak and mean gradient for patients with an aortic prosthesis were 26 and $15 \mathrm{~mm} \mathrm{Hg}$, respectively, and the 90th percentile values for peak and mean aortic gradients were 50 and $29 \mathrm{~mm} \mathrm{Hg}$, respectively. Aortic peak and mean gradients were significantly higher in patients with mismatch defined by using any 1 of the 3 definitions examined in the multivariate models. For instance, in patients with an aortic iEOA less than or equal to $0.80 \mathrm{~cm}^{2} / \mathrm{m}^{2}$, the median peak and mean aortic gradients were 29 and $16 \mathrm{~mm} \mathrm{Hg}$, respectively, versus 26 and $14 \mathrm{~mm}$ $\mathrm{Hg}$, respectively, in those without mismatch $(P<.002)$. Compared with those with an iEOA greater than $0.80 \mathrm{~cm}^{2} /$ $\mathrm{m}^{2}$, patients with an aortic iEOA less than or equal to 0.80 $\mathrm{cm}^{2} / \mathrm{m}^{2}$ had a 1.79 relative risk of having an aortic peak transprosthesis gradient at or greater than the cohort's 90th percentile value $(P=.01)$.

Peak and mean transprosthesis gradients had a significant negative effect on freedom from heart failure after valve replacement, both as a linear relationship (HR, 1.03 and 1.06 per millimeter increase in peak and mean gradient, respectively; $P<.001$ ) and as a dichotomous predictor defined by a gradient at or more than the 90th percentile value of the cohort (HR, 2.5 and 2.8 for increased peak and mean gradients, respectively; $P \leq .005$ ).

\section{Risk Factors for All-Cause Death}

Table 4 displays risk factors for all-cause death late after aortic valve replacement in the study cohort. Significant independent predictors were age, atrial fibrillation, preoperative functional class, left ventricular grade, pulmonary artery diastolic pressures, coronary artery disease, the number of pack-years smoked, and insulin-dependent diabetes. Although a trend in predicting all-cause mortality was observed for EOA (HR, 0.4 per square centimeter increase; $P$ $=.09$ ) and prosthesis-patient mismatch defined as EOA/ BSA less than or equal to $0.80 \mathrm{~cm}^{2} / \mathrm{m}^{2}$ (HR, 1.4 vs patients with EOA/BSA $\left.>0.80 \mathrm{~cm}^{2} / \mathrm{m}^{2} ; P=.15\right)$, no significant 
independent effect of prosthesis size on crude survival was demonstrated.

\section{Discussion}

This study identified risk factors for persistent or recurrent heart failure after aortic valve replacement from a cohort of patients who were prospectively followed up after surgery. Risk factors for the composite outcome of heart failure symptoms or death from heart failure after aortic valve replacement were age older than 65 years, atrial fibrillation, advanced preoperative NYHA class, increased preoperative diastolic pulmonary artery pressures, left ventricular grade, coronary artery disease, smoking, redo aortic valve replacement, concomitant tricuspid insufficiency, smaller prosthesis size, postoperative transprosthesis gradient, and prosthesis-patient mismatch defined as either iEOA less than or equal to $0.75 \mathrm{~cm}^{2} / \mathrm{m}^{2}$ or iEOA less than or equal to 0.80 $\mathrm{cm}^{2} / \mathrm{m}^{2}$, which resulted in an increase in estimated heart failure risk of approximately $60 \%$. There was also a trend for prosthesis-patient mismatch defined as iEOA less than or equal to $0.75 \mathrm{~cm}^{2} / \mathrm{m}^{2}$ to predict a decreased freedom from the specific hard end point of CHF death after valve replacement. However, prosthesis-patient mismatch in its classic definition, ie, iEOA less than or equal to $0.85 \mathrm{~cm}^{2} / \mathrm{m}^{2}$, was not associated with an increased incidence of CHF symptoms, CHF death, or their combined occurrence. Valve size and mismatch indicators also significantly predicted the cumulative incidence of heart failure at 1 and 5 years after aortic valve replacement, at which time patients in mismatch had twice the odds of having had heart failure events compared with those not in mismatch, but not at 10 years, where it is possible that competing risks related to bioprosthesis structural deterioration, progression of underlying left ventricular dysfunction, or development of coronary artery disease may have diminished the overall effect of prosthesis size and mismatch on freedom from CHF.

Prosthesis size and mismatch were not found to be independently predictive of the incidence of all-cause death in this study. Despite all-cause mortality being a robust and easily interpretable end point, it is limited as a specific indicator by the plethora of confounding and contributing factors and by the availability of medical therapy for the palliation of mild to moderate heart failure. In an elderly patient population, such as in this cohort, competing causes of death such as coronary disease, valve-related complications, cancer, and others may also have diluted the effect of patient-prosthesis mismatch on all-cause mortality after aortic valve replacement. A primary end point of CHF was therefore chosen a priori as a more specific indicator of long-term outcome after valve replacement, because CHF is a relevant clinical condition that translates into decreased quality of life for the patient and directly reflects on the success of valve replacement.

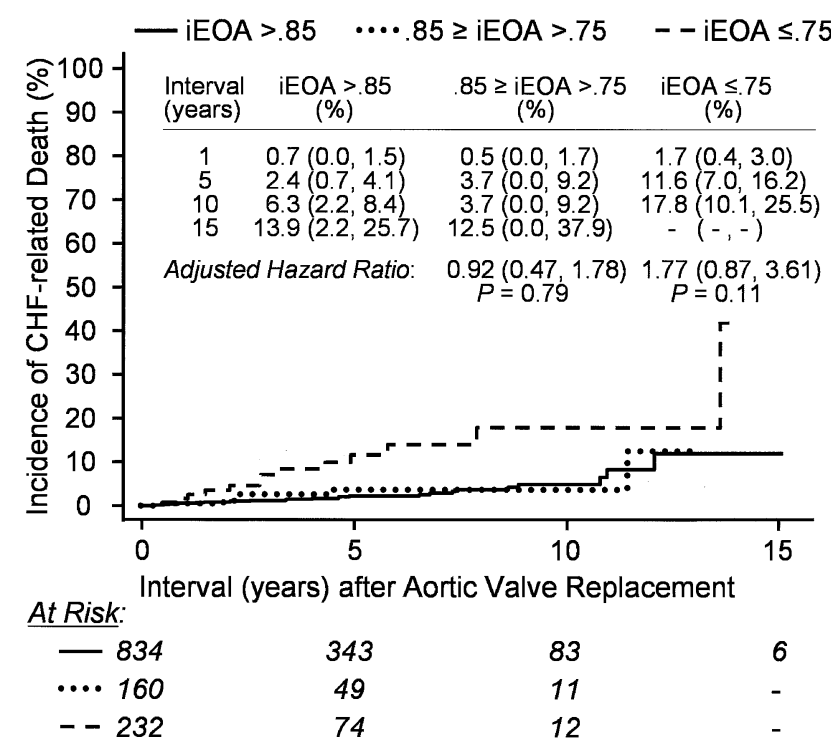

Figure 2. Effect of prosthesis-patient mismatch on the incidence of CHF-related death after aortic valve replacement, adjusted for age, preoperative NYHA class, smoking, atrial fibrillation, and coronary disease. Patients with an indexed effective orifice area (iEOA) between 0.75 and $0.85 \mathrm{~cm}^{2} / \mathrm{m}^{2}$ had an equivalent incidence of CHF-related death compared with those with an IEOA of more than $0.85 \mathrm{~cm}^{2} / \mathrm{m}^{2}$; however, a trend toward an increased risk of CHF death was observed in patients with an IEOA equal to or less than $0.75 \mathrm{~cm}^{2} / \mathrm{m}^{2}$.

Several of the risk factors for CHF after valve replacement identified in this study have heightened relevance in that they are potentially modifiable. One patient-related factor is smoking, and although the possible benefits of smoking cessation in valve patients are only suggested by the results of this study, for this reason as well as for other health benefits, patients should be strongly encouraged to refrain from smoking after valve replacement. Other modifiable risk factors relate to clinical decision making and surgical technique, because advanced preoperative NYHA functional class before operation, persistent tricuspid regurgitation after surgery, chronic atrial fibrillation, small prosthesis size, and prosthesis-patient mismatch (iEOA $\leq 0.80$ $\mathrm{cm}^{2} / \mathrm{m}^{2}$ ) were identified as independent predictors of heart failure after valve replacement. It is possible, although undetermined from these data, that current trends in the treatment of patients with valve disease-such as the referral of patients for valve repair or replacement at an earlier symptomatic stage before left ventricular dysfunction occurs, ${ }^{1}$ the renewed emphasis on the concomitant surgical treatment of tricuspid valve insufficiency during left heart valve operations, ${ }^{15}$ the expanded indications for concomitant atrial fibrillation surgery, ${ }^{16}$ and the avoidance of aortic prosthesis-patient mismatch by the selective use of stentless valves, homografts, and root enlargement procedures ${ }^{13}$ 
may have a beneficial effect on freedom from heart failure after aortic valve replacement.

Whether aortic prosthesis-patient mismatch is relevant or not has been a subject of intense controversy in the cardiac literature. One inherent difficulty in answering this question relates to the fact that surgical decision making and confounding by indication ${ }^{17}$ may bias against patients with small aortic prostheses with respect to operative mortality, all-cause survival, and long-term functional symptoms. ${ }^{9}$ In this situation, the surgeon's choice of prosthesis type and size during valve replacement is heavily influenced by the perceived balance between the increased perioperative risk of the more complex operation needed to avoid a potentially suboptimal late clinical outcome due to a small prosthesis, and the chances of the patient experiencing meaningful long-term survival and quality of life. Perioperative outcomes may be particularly susceptible to confounding by indication, and consequently this study focused on functional cardiac outcomes in patients who survived aortic valve replacement. Confounding was also reduced by the use of bootstrapped regression models in which multiple potential confounders were simultaneously accounted for, regardless of their $P$ value on univariate analysis.

Our findings support the concept that there is a minimal threshold in prosthesis size related to the patient's BSA, somewhere near an iEOA of 0.75 or $0.80 \mathrm{~cm}^{2} / \mathrm{m}^{2}$, beyond which optimal cardiac recovery is less likely to occur after valve replacement. The concept of prosthesis-patient mismatch was first introduced by Rahimtoola ${ }^{18}$ in 1978 as a condition in which the in vivo prosthetic valve EOA is less than that of the native human valve. The physiologic sequelae of mismatch were subsequently proposed to relate to persistent valve gradients on the basis of in vitro studies conducted by Dumesnil and Yoganathan, ${ }^{19}$ who demonstrated an exponential relationship between mean transprosthetic pressure gradient and iEOAs for aortic bioprostheses in an in vitro physiologic pulse-duplicator system. Their results fostered the recommendation that iEOA should ideally not be less than 0.9 to $1 \mathrm{~cm}^{2} / \mathrm{m}^{2}$ for aortic bioprostheses to minimize residual postoperative transprosthetic peak pressure gradients and led to the premise that there may be a correlation between the decrease in transvalvular gradient and the clinical improvement seen after surgery. ${ }^{20,21}$

Determining mismatch on the basis of postoperative echocardiographic gradients may, however, be flawed, because it may not adequately take into account patients in whom persistent left ventricular dysfunction due to the intrinsically stenotic valve may perpetuate a decreased cardiac output situation with failure to generate the expected high transprosthetic gradients. ${ }^{22}$ This limitation may explain previous data reported by Hanayama and associates, ${ }^{23}$ who did not demonstrate a difference in clinical outcome in patients with high transprosthesis gradients after aortic valve replacement. Although our study examined the effect of postoperative gradient and found transprosthesis gradients to be both predicted by patient-prosthesis mismatch and predictive of a decreased freedom from postoperative heart failure, postoperative gradients may constitute a less than optimal method to define prosthesis-patient mismatch, because gradients are measured after valve replacement has taken place and may be unreliable in the setting of persistent left ventricular dysfunction.

Work from Medalion and colleagues ${ }^{8}$ has indicated that small prosthesis size is not a predictor of long-term outcome, and other authors have also failed to show a correlation between mismatch, early or late all-cause mortality, and morbidity. ${ }^{24-26}$ In the studies of Knez and associates ${ }^{24}$ and Medalion and associates, ${ }^{8}$ outcomes were, however, correlated with indexed measures of the geometric internal orifice area (GIOA). The GIOA was derived on the basis of the internal area calculated from the documented internal valve orifice diameter. The documented internal valve orifice diameter either was provided from the manufacturer or was measured by using microcalipers. ${ }^{12}$ GIOA is, however, a 2-dimensional measure that does not take into account the other characteristics of a valve that may contribute significantly to minimizing the effective valve orifice, such as valve height and leaflet structures within the valve. For these reasons, the in vivo EOA derived from patients with normally functioning valves is superior and more clinically relevant. ${ }^{13}$ Differences between GIOA and EOA may also explain why there is such a discrepancy in the incidence of prosthesis-patient mismatch among many of the available studies, because previous studies have shown that the ratio between the GIOA and the EOA may significantly vary depending on the type of prosthesis. ${ }^{27,28}$ In a study from Pibarot and colleagues, ${ }^{29}$ the incidence of mismatch was $71 \%$ for stented prostheses and 29\% for stentless prostheses, more within the range observed in our population, with an incidence of $32 \%$, and in contrast to the study from Medalion and colleagues, ${ }^{8}$ in which mismatch was present in less than $1 \%$ of patients with a calculated GIOA threshold of less than $0.85 \mathrm{~cm}^{2} / \mathrm{m}^{2}$.

\section{Selection Bias and Confounding}

This study was not randomized, and it is possible that despite the use of specific CHF end points and multivariate bootstrapped methods, confounding by indication, selection bias, or unidentified confounders may have influenced the results, especially in patients in whom a smaller aortic prosthesis was implanted.

\section{Censoring and Follow-up}

The Kaplan-Meier and Cox proportional hazard methods require the implicit assumption that censoring is independent of clinical outcomes, which cannot be verified. It is 
possible that patients lost to follow-up may have had outcomes that were important but not accounted for in the analyses or that resulted in their being lost to follow-up. Furthermore, the median follow-up of the study, although it extended to 17.1 years, was only 4.3 years. Inferences therefore apply mostly to intermediate-term outcomes.

\section{Missing Entries}

The database contained incomplete entries, for instance, with respect to echocardiographic data. Missing data were not imputed, and the sample size of each analysis corresponded to the number of complete entries; this approach may, however, have prevented the demonstration of some associations by increasing type II statistical error, because potential predictors were not all evaluated by using total samples.

\section{Effective Orifice Area}

This study used internal aortic diameters and in vivo EOAs as primary measures of valve size after aortic valve replacement. This is likely not as accurate as the actual measurements of prosthesis EOA for each patient after operation, which were not routinely available in the cohort, but the latter are also more susceptible to errors if low cardiac output persists after valve replacement.

\section{Inference and Generalizability}

This study used a relatively specific composite outcome based on NYHA class and CHF mortality, but it did not examine all outcomes that may relate to cardiac performance and functional status after valve replacement. No data were routinely available from the cohort with respect to exercise tolerance measured by standardized protocols, left ventricular mass regression, or left ventricular performance during exercise. Furthermore, to use a composite outcome of CHF death or symptoms, the cumulative incidence rather than the prevalence of heart failure was used in the study, which may have resulted in a seemingly high percentage of symptomatic patients compared with a prevalence-based outcome and does not provide an estimate of the duration of symptoms in affected patients, some of whom may have experienced durable symptom resolution with medical management alone.

The findings of this study, like those of other large observational cohorts, may not necessarily be generalizable to all patients with prosthetic valves, because the study represents a single institution's experience, is not randomized, and may have been affected by regional referral and patient treatment patterns. Unsuspected demographic and selection factors unique to the study cohort may therefore have resulted in overfitted statistical associations. Statistical overfitting was, however, made less likely by the use of bootstrapped simulations.

\section{Conclusions}

This study identified risk factors for the development of heart failure after aortic valve replacement. Potentially modifiable factors, such as advanced NYHA class, left ventricular grade, and increased preoperative pulmonary diastolic pressures, may be affected by earlier referral for valve operation. Other risk factors, such as postoperative tricuspid insufficiency and atrial fibrillation, warrant further research to better define the effect of concomitant surgical procedures oriented at these problems on the freedom from $\mathrm{CHF}$ after valve replacement. The findings of this study also suggest that the implantation of an aortic valve prosthesis with an estimated iEOA equal to or less than $0.80 \mathrm{~cm}^{2} / \mathrm{m}^{2}$ should be avoided whenever feasible, because this results in a estimated increase in the risk of heart failure after valve replacement of approximately $60 \%$, despite not significantly affecting crude long-term survival.

The authors thank Mary Thomson for her assistance with the organization of the valve clinic and the management of the database.

\section{References}

1. ACC/AHA guidelines for the management of patients with valvular heart disease. A report of the American College of Cardiology/American Heart Association. Task Force on Practice Guidelines (Committee on Management of Patients with Valvular Heart Disease). J Am Coll Cardiol. 1998;32:1486-588.

2. Manouguian S, Seybold-Epting W. Patch enlargement of the aortic valve ring by extending the aortic incision into the anterior mitral leaflet. New operative technique. J Thorac Cardiovasc Surg. 1979;78: 402-12.

3. Urbanski PP. Complete aortic root replacement in patients with small aortic annulus. Ann Thorac Surg. 2002;73:725-8 ; discussion 728-9.

4. Dellgren G, Feindel CM, Bos J, et al. Aortic valve replacement with the Toronto SPV: long-term clinical and hemodynamic results. Eur J Cardiothorac Surg. 2002;21:698-702.

5. Rajappan K, Melina G, Bellenger NG, et al. Evaluation of left ventricular function and mass after Medtronic Freestyle versus homograft aortic root replacement using cardiovascular magnetic resonance. J Heart Valve Dis. 2002;11:60-5; discussion 66.

6. Sintek CF, Fletcher AD, Khonsari S. Stentless porcine aortic root: valve of choice for the elderly patient with small aortic root? J Thorac Cardiovasc Surg. 1995;109:871-6; discussion 876.

7. Sommers KE, David TE. Aortic valve replacement with patch enlargement of the aortic annulus. Ann Thorac Surg. 1997;63:1608-12.

8. Medalion B, Blackstone EH, Lytle BW, et al. Aortic valve replacement: is valve size important? J Thorac Cardiovasc Surg. 2000;119: 963-74.

9. Rao V, Jamieson WR, Ivanov J, et al. Prosthesis-patient mismatch affects survival after aortic valve replacement. Circulation. 2000;102:III5-9.

10. Edmunds LH Jr, Cohn LH, Weisel RD. Guidelines for reporting morbidity and mortality after cardiac valvular operations. $J$ Thorac Cardiovasc Surg. 1988;96:351-3.

11. Edmunds LH Jr, Clark RE, Cohn LH, et al. Guidelines for reporting morbidity and mortality after cardiac valvular operations. The American Association for Thoracic Surgery, Ad Hoc Liason Committee for Standardizing Definitions of Prosthetic Heart Valve Morbidity. Ann Thorac Surg. 1996;62:932-5.

12. Christakis GT, Buth KJ, Goldman BS, et al. Inaccurate and misleading valve sizing: a proposed standard for valve size nomenclature. Ann Thorac Surg. 1998;66:1198-203.

13. Pibarot P, Dumesnil JG. Hemodynamic and clinical impact of pros- 
thesis-patient mismatch in the aortic valve position and its prevention J Am Coll Cardiol. 2000;36:1131-41.

14. Efron B, Tibshirani R. Bootstrap methods for standard errors, confidence intervals, and other measures of statistical accuracy. Stat Sci. 1986;1:54-77.

15. Gatti G, Maffei G, Lusa AM, Pugliese P. Tricuspid valve repair with the Cosgrove-Edwards annuloplasty system: early clinical and echocardiographic results. Ann Thorac Surg. 2001;72:764-7.

16. Gillinov AM, Blackstone EH, McCarthy PM. Atrial fibrillation: current surgical options and their assessment. Ann Thorac Surg. 2002;74: 2210-7.

17. Rosendaal FR. Bridging case-control studies and randomized trials. Curr Control Trials Cardiovasc Med. 2001;2:109-10.

18. Rahimtoola SH. The problem of valve prosthesis-patient mismatch. Circulation. 1978;58:20-4.

19. Dumesnil JG, Yoganathan AP. Valve prosthesis hemodynamics and the problem of high transprosthetic pressure gradients. Eur J Cardiothorac Surg. 1992;6(suppl 1):S34-7 ; discussion S38.

20. Gonzalez-Juanatey JR, Garcia-Acuna JM, Vega Fernandez M, et al. Influence of the size of aortic valve prostheses on hemodynamics and change in left ventricular mass: implications for the surgical management of aortic stenosis. J Thorac Cardiovasc Surg. 1996;112:273-80.

21. Pibarot P, Honos GN, Durand LG, Dumesnil JG. The effect of prosthesis-patient mismatch on aortic bioprosthetic valve hemodynamic performance and patient clinical status. Can J Cardiol. 1996;12:37987.

22. Burwash IG, Hay KM, Chan KL. Hemodynamic stability of valve area, valve resistance, and stroke work loss in aortic stenosis: a comparative analysis. J Am Soc Echocardiogr. 2002;15:814-22.

23. Hanayama N, Christakis GT, Mallidi HR, et al. Patient prosthesis mismatch is rare after aortic valve replacement: valve size may be irrelevant. Ann Thorac Surg. 2002;73:1822-9 ; discussion 1829.

24. Knez I, Rienmuller R, Maier R, et al. Left ventricular architecture after valve replacement due to critical aortic stenosis: an approach to dis-/ qualify the myth of valve prosthesis-patient mismatch? Eur J Cardiothorac Surg. 2001;19:797-805.

25. Fernandez J, Chen C, Laub GW, et al. Predictive value of prosthetic valve area index for early and late clinical results after valve replacement with the St Jude Medical valve prosthesis. Circulation. 1996;94: II109-12.

26. Knez I, Machler H, Rehak P, et al. Concomitant procedures in the small versus standard aortic root. J Heart Valve Dis. 1996;5(suppl 3):S294-301

27. Yoganathan AP, Woo YR, Sung HW, et al. In vitro hemodynamic characteristics of tissue bioprostheses in the aortic position. $J$ Thorac Cardiovasc Surg. 1986;92:198-209.

28. Baumgartner H, Khan SS, DeRobertis M, et al. Doppler assessment of prosthetic valve orifice area. An in vitro study. Circulation. 1992;85: 2275-83.

29. Pibarot P, Dumesnil JG, Cartier PC, et al. Patient-prosthesis mismatch can be predicted at the time of operation. Ann Thorac Surg. 2001;71: S265-8.

\section{Discussion}

Dr A. Marc Gillinov (Cleveland, Ohio). Dr Ruel and colleagues are to be congratulated for this excellent study, which represents an enormous body of work. There is considerable controversy concerning the impact of prosthesis size in patients receiving aortic valve replacement, and I will confine my comments to the aortic valve patients.

We would all like to believe that a larger prosthesis results in lesser gradients, a greater reduction of left ventricular mass, and better clinical outcomes, but several recent studies suggest that the impact of size in modern and hemodynamically efficient prostheses is limited.

In their assessment of functional outcomes after aortic valve replacement, Dr Ruel and colleagues identify several risk factors for CHF. They found that smaller prosthesis size, particularly when expressed in terms of EOA, was independently associated with CHF. Although this relationship was generally true at 1 and 5 years after surgery, it was not true at 10 years. I have several questions related to the method and the conclusions.

First, with respect to patients receiving double valve replacement, both aortic and mitral valves, these patients with 2 affected left-sided valves may have a worse prognosis than patients with only an aortic valve replacement. Were patients having only an aortic valve analyzed separately?

Second, with respect to measures of valve size and patient size, aortic valve size can be expressed by geometric measures such as internal orifice diameter and by functional measures such as EOA. These methods provide somewhat different information, and each can be indexed to patient size. The EOAs that you used for analysis were derived from published figures rather than your own patients in whom you did have echocardiographic data. Why did you use published figures rather than your own echocardiographic data, and, in addition, did you index the internal orifice diameter, which is a geometric dimension that does not change, in your patients?

What were the sizes of the valves implanted? How many people received valves of labeled size 19 and 21? How common was prosthesis-patient mismatch if you measure it and call it to be less than $.75 \mathrm{~cm}^{2} / \mathrm{m}^{2}$ ?

I congratulate you and your coauthors on this ambitious study, which I think is just filled with useful clinical information.

Dr Ruel. Thank you, Dr Gillinov, for your excellent points. In response to your first question, you are entirely right in that patients with double valve replacement were not excluded from the cohort, which therefore included patients who received aortic valve replacement alone and those who received double valve replacement. There was no significant difference in the freedom from $\mathrm{CHF}$ between the aortic valve replacement and the aortic valve replacement plus mitral valve replacement subgroups. In addition, co-presence of a mitral prosthesis in aortic valve replacement patients was added as one of the multivariate predictors and was accounted for in the models. (NOTE FROM THE AUTHORS: Subsequent to this discussion, the analyses and results reported in this article pertain specifically to patients who had replacement of the aortic valve alone.)

To answer your second question, measures of prosthesis size examined in this study had an effect both as an absolute measurement and after being indexed for BSA. In other words, their impact was significant both as an absolute measure and after indexing for BSA.

Regarding your point on the use of EOAs, you are absolutely right in that the values used were the published values, supplemented with data from the valve manufacturer. Although there are limitations to this methodology, because one is not actually measuring the individual EOA of each patient, we believe that there are several advantages. First, our use of EOA is preferred over the geometric internal valve area, derived from a simple mathematical equation that uses the internal diameter of the prosthesis, because the former measures the physiologic flow area of the prosthesis. Second, the determination of an individual's EOA by using Doppler echocardiography requires an accurate measurement of the left ventricular outflow tract diameter, outflow tract velocity, and 
transvalvular velocity. These can be difficult to obtain in some patients because of their body characteristics or because of artifacts produced by the prosthesis itself. Our approach is advantageous in that it provides data that can be used clinically to modify the operative strategy at the time of valve implantation to prevent patient-prosthesis mismatch.

Coming to your last question with respect to the incidence of small valves, approximately $2 \%$ of the cohort's patients received valves of size $19 \mathrm{~mm}$. This increased to approximately $24 \%$ of patients who received either a size 19 or a size 21 aortic valve. Prosthesis-patient mismatch defined as $0.80 \mathrm{~cm}^{2} / \mathrm{m}^{2}$ was prevalent in approximately $26 \%$ of the study population. So, at least in our practice, mismatch does appear to constitute a significant problem, since more than one quarter of patients could potentially be prevented from presenting this risk factor for CHF events after aortic valve replacement. I would like to thank you for your excellent questions and comments.

Dr Hormoz Azar (Norfolk, Va). Excellent study: congratulations. Considering that patients who had mismatch generally had more severe aortic stenosis, the question is, for the increased failure, how much of it was related to previous ventricular fibrosis and diastolic dysfunction, and how much of it was really related to the prosthesis mismatch? Did you try to look at the wall thickness and septal thickness and correlate that in any way?

Dr Ruel. Thank you for your excellent question. Unless there was strong collinearity, we did not formally ascertain whether patients who had mismatch also had a higher preoperative prevalence of other predictive factors, such as poor left ventricular grade. We do not know whether a biologic correlation exists between having a small aortic annulus and having more left ventricular dysfunction prior to valve replacement. However, an array of potential confounding factors was accounted for in the multivariate models, so the impact reported for mismatch or small valve size is independent of confounders such as preoperative NYHA class, preoperative left ventricular grade, pulmonary pressures, and so on. However, possible correlations between mismatch and myocardial thickness measurements after surgery were not formally examined.

Access to The Journal of Thoracic and Cardiovascular Surgery Online is reserved for print subscribers!

Full-text access to The Journal of Thoracic and Cardiovascular Surgery Online is available for all print subscribers. To activate your individual online subscription, please visit The Journal of Thoracic and Cardiovascular Surgery Online, point your browser to http://www.mosby.com/jtcvs, follow the prompts to activate your online access, and follow the instructions. To activate your account, you will need your subscriber account number, which you can find on your mailing label (note: the number of digits in your subscriber account number varies from 6 to 10 ). See the example below in which the subscriber account number has been circled:

\section{Sample mailing label}

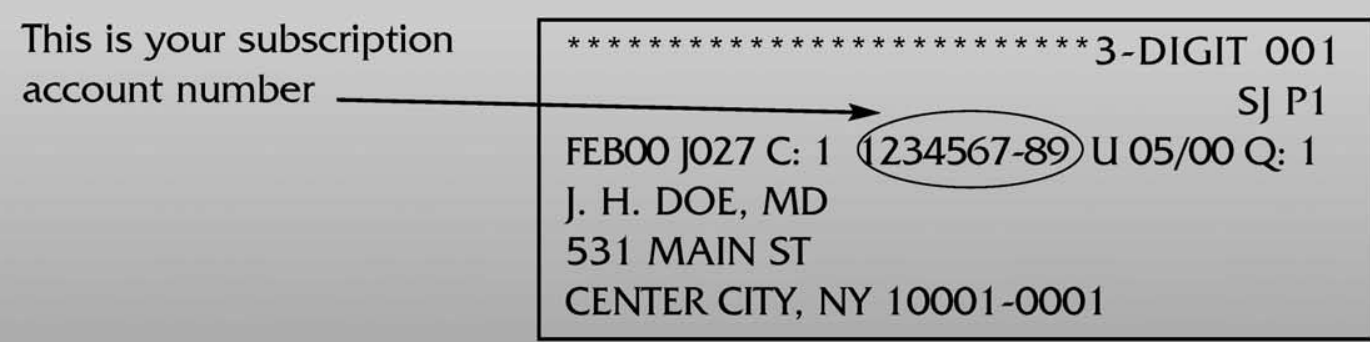

Personal subscriptions to The Journal of Thoracic and Cardiovascular Surgery Online are for individual use only and may not be transferred. Use of The Journal of Thoracic and Cardiovascular Surgery Online is subject to agreement to the terms and conditions as indicated online. 